\title{
Safety and efficacy of bendamustine in the conditioning regimen for autologous stem cell transplantation in patients with relapsed/refractory lymphoma
}

\author{
Munira Shabbir-Moosajee ${ }^{1}$, Samad Jehangir ${ }^{2}$, Sobiya Sawani ${ }^{3}$, Tariq Muhammed ${ }^{2}$, Natasha $\mathrm{Ali}^{4}$, \\ Usman Sheikh ${ }^{4}$, Salman Adil ${ }^{4}$ \\ ${ }^{1}$ Hematology/Oncology and Bone Marrow Transplantation, ${ }^{2}$ Department of Oncology, Aga Khan University Hospital, ${ }^{3}$ Department \\ of Community Health Sciences, ${ }^{4}$ Hematology and Laboratory Medicine, Aga Khan University Hospital, Karachi, Pakistan
}

p-ISSN 2287-979X / e-ISSN 2288-0011 https://doi.org/10.5045/br.2019.54.2.108 Blood Res 2019;54:108-113.

Received on October 31, 2018 Revised on December 18, 2018 Accepted on December 26, 2018

\author{
Correspondence to \\ Munira Shabbir-Moosajee, M.D. \\ Hematology/Oncology and Bone Marrow \\ Transplantation, Aga Khan University \\ Hospital, Karachi 74800, Pakistan \\ E-mail: munira.moosajee@aku.edu \\ (C) 2019 Korean Society of Hematology
}

\begin{abstract}
Background
Bendamustine is an attractive option for the management of both de novo and relapsed lymphomas. It is being increasingly used in the conditioning regimen for autologous stem cell transplantation (SCT) and can be an alternative to the traditionally-used carmustine. In this study, we aimed to determine the safety and efficacy of bendamustine in the conditioning regimen for autologous SCT in refractory/relapsed lymphomas.
\end{abstract}

\section{Methods}

We designed a descriptive study to evaluate bendamustine in combination with etoposide, cytarabine, and melphalan (BeEAM) in the conditioning regimen for autologous SCT.

Results

Fourteen patients (median age, $28 \mathrm{yr}$ ) with Hodgkin's lymphoma $(\mathrm{HL})(\mathrm{N}=8)$, non-Hodgkin's lymphomas $(\mathrm{NHL})(\mathrm{N}=5)$, or peripheral T-cell lymphoma, not otherwise specified (PTCL NOS) $(N=1)$ were included in the study. A median number of $5.95 \times 10^{6}$ $\mathrm{CD} 34+$ cells/kg were transfused. Median times to absolute neutrophil count and platelet engraftment were 17 days and 24 days, respectively. The 100-day transplantation mortality rate was $28 \%$ (4 patients). Eight patients $(57.14 \%$ ) had GII-III acute kidney injury, four patients $(28.5 \%)$ had GIII-IV hyperbilirubinemia, and twelve patients (85\%) had GII-III diarrhea. After 3 months, 37\% (5 patients) and 21.4\% (3 patients) demonstrated complete response and partial response, respectively. The median follow-up was 5.5 months (15 days-19 mo). At the final follow-up, 7 patients (50\%) were alive and in CR.

\section{Conclusion}

Our study showed that bendamustine is a potentially toxic agent in the conditioning regimen for autologous SCT, resulting in significant liver, kidney, and gastrointestinal toxicity. Further studies are required to assess its safety and efficacy at reduced doses.

Key Words Bendamustine, Toxicity, Autologous stem cell transplant, Lymphoma

\section{INTRODUCTION}

Hodgkin's lymphoma (HL) and non-Hodgkin's lymphomas (NHL) are highly chemotherapy-sensitive tumors. However, there is a significant subset of patients who relapse or who never achieve complete remission. Second line salvage chemotherapy has been shown to be very effective in patients with chemosensitive diseases with CR rates great- er than $50 \%$, as observed with aggressive second line chemo immunotherapy in relapsed diffuse large B cell lymphoma (DLBCL) [1, 2].

However, the prognosis is usually poor with conventional salvage regimens alone $[3,4]$. High dose therapy (HDT) followed by stem cell rescue is the treatment of choice for patients with relapsed/refractory lymphoma. Philip et al. [5] demonstrated a statistically significant difference in 5-year event-free survival ( $46 \%$ vs. $12 \% ; P=0.001)$ and OS 
(53\% vs. 32\%; $P=0.038$ ) for patients undergoing autologous stem cell transplantation (ASCT) and salvage chemotherapy, respectively. With improvement in supportive care and management of toxicity resulting from the conditioning regimen, the survival rates after ASCT are approaching 60\% for B cell lymphomas [6] and $40-70 \%$ in patients with HL [7, 8].

Carmustine, etoposide, and cytarabine with melphalan (BEAM) is the most common conditioning regimen used for ASCT for relapsed lymphoma. Other regimens that have been studied include cyclophosphamide (BEAC), cyclophosphamide, carmustine, and etoposide (CBV), and cyclophosphamide-TBI (total body irradiation), but BEAM remains the preferred conditioning regimen $[9,10]$. Recently, availability of carmustine has been problematic in certain parts of the world. Therefore, there is increasing interest in exploring other active agents that could potentially replace carmustine in the conditioning regimen for ASCT. Bendamustine is an attractive option to replace carmustine because it combines the alkylating activity of the nitrogen mustard group with the antimetabolite activity of the purine analog structure (benzimidazole ring). Given the uniqueness of its chemical structure, it has a different mechanism of action from traditional alkylating agents. Carmustine demonstrates only partial cross-resistance (in vitro) with other alkylating agents. It leads to cell death via single and double strand DNA cross-linking, and has also been shown to activate apoptotic pathways [11]. Therefore, bendamustine has clinical potential for treating diseases that are refractory to alkylators. It has shown superior efficacy compared to standard therapies for relapsed chronic lymphocytic leukemia and indolent NHL [12], and is considered a standard first line treatment for indolent B cell lymphoma [13]. These data make it an attractive option as part of the conditioning regimen in ASCT.

Carmustine is not available in Pakistan. After encouraging results from a study by Visani et al. [14] demonstrating efficacy and manageable toxicity of cotreatment of bendamustine with etoposide, cytarabine, and melphalan, we have been exclusively using bendamustine, cytarabine, etoposide, and carmustine (BeEAM) as the conditioning regimen for ASCT to treat relapsed/refractory lymphoma at our institution. Here, we present the safety and efficacy data from use of the BeEAM protocol from the last 2 years.

\section{MATERIALS AND METHODS}

\section{Patient population}

Fourteen patients with resistant/relapsed lymphomas, including both NHL and HL, were included in this study. Patient files were retrospectively reviewed to collect data regarding their diseases, treatments, toxicities, and outcomes. This protocol was approved by the Ethical Review Committee of Aga Khan Hospital, Karachi.

\section{Mobilization and conditioning regimen}

Patients were mobilized with granulocyte colony stimulating factor (GCSF) (5 mg/kg, twice per day subcutaneously for 4 days) or cyclophosphamide. Patients that received cyclophosphamide $\left(1.5 \mathrm{~g} / \mathrm{m}^{2}\right)$ then received GCSF $(5 \mathrm{mg} / \mathrm{kg}$, twice daily subcutaneously for 6 days). Patients that underwent mobilization with GCSF alone underwent scheduled stem cell pheresis on day 4. For patients who received cyclophosphamide and GCSF mobilization, stem cells were harvested on days 5 to 7 (depending on count recovery) using either a Cobe Spectra AutoPBSC or a COMTEC (Fresenius Kabi, Bad Homberg, Germany) system in the default software configuration recommended by the manufacturer. After stem cell collection, patients received the conditioning regimen, which consisted of bendamustine coupled with fixed doses of etoposide, cytarabine, and melphalan (BeAM) administered in an inpatient setting. BeAM regimen was given per standard protocols and institutional guidelines. Hyperhydration was started on day -6 , followed by bendamustine $150 \mathrm{mg} / \mathrm{m}^{2}$ (days -5 and -4 ), etoposide $150 \mathrm{mg} / \mathrm{m}^{2}$ (days -5 to -2 ), cytarabine $150 \mathrm{mg} / \mathrm{m}^{2}$ (days -5 to -2 ), and melphalan $75 \mathrm{mg} / \mathrm{m}^{2}$ (day -1). Stem cell infusion was performed on day 0 . All patients received granulocyte colony stimulating factor (GCSF) from day +1 .

\section{Supportive care and monitoring}

A central line was inserted in all patients. They were admitted to a single room, and reverse barrier nursing was practiced. All patients were given antimicrobials (levofloxacin $500 \mathrm{mg}$ od or ciprofloxacin $500 \mathrm{mg}$ bid), an antifungal (voriconazole $200 \mathrm{mg} \mathrm{qd}$ ), and an antiviral (valacyclovir 500 $\mathrm{mg}$ bid) as prophylaxis starting at day -5 . Blood tests were performed per standard protocols, and blood products were transfused to keep hemoglobin levels $>8 \mathrm{~g} / \mathrm{dL}$ and platelet count $>15 \times 10^{9}$. In cases in which fever developed, patients were given intravenous broad-spectrum antibiotics. Piperacillin tazobactam or imipenem/meropenem were given for gram negative bacilli coverage. Vancomycin was the drug of choice for management of gram positive cocci. In addition, amphotericin B was used when fungal infections were suspected. Patient symptoms were assessed daily, and physical examinations were administered during their hospital stay and as per recommendations after follow-up.

\section{Response criteria}

Engraftment was defined as achievement of an absolute neutrophil count of $0.5 \times 10^{9}$ per liter for 3 consecutive days. Platelet engraftment was defined as a count of $20 \times 10^{9}$ per liter with transfusion independence. Patients were evaluated for response at 4 weeks after transplant, and then at day 100, at the outpatient clinic. At subsequent follow-up visits, imaging was performed per the discretion of the treating physicians. All toxicities were defined using the National Cancer Institute's Common Terminology Criteria for Adverse Events Version 3.0 (2003). All patients were evaluated using either computed tomography (CT) or positron emission tomography (PET) before and after ASCT. 


\section{Statistical analysis}

The primary end point was to assess the safety and efficacy of bendamustine in the conditioning regimen for autologous stem cell transplantation in patients with relapsed/refractory lymphoma. The secondary objectives included assessment of time to progression and overall survival. Statistical analysis was performed in accordance with approved protocols. The Kaplan-Meier method was used to estimate overall survival. Data were analyzed using Strata 12.1 software.

\section{RESULTS}

\section{Patient characteristics}

Patient characteristics are summarized in Table 1. Fourteen patients were included in this study. The median age of the included patients was 28 years (range, 16-58 yr). Eleven $(78.5 \%)$ patients were males and three $(21.43 \%)$ patients were females. Eight patients (57.14) had Hodgkin's Lymphoma, five patients (35.71\%) had Diffuse Large B cell Lymphoma, and one patient (7.14\%) had peripheral T-cell lymphoma, not otherwise specified (PTCL NOS). DHAP/

Table 1. Patient characteristics.

\begin{tabular}{|c|c|c|}
\hline Characteristics & $\mathrm{N}$ & $\%$ \\
\hline Age, median & 14 & $28 \mathrm{yr}$ \\
\hline \multicolumn{3}{|l|}{ Sex } \\
\hline Male & 11 & 78.57 \\
\hline Female & 3 & 21.43 \\
\hline BMI, median & 14 & 25.5 \\
\hline \multicolumn{3}{|l|}{ IPI } \\
\hline 0 & 3 & 21.4 \\
\hline 1 & 6 & 42.8 \\
\hline 2 & 2 & 14.2 \\
\hline 3 & 3 & 21.4 \\
\hline \multicolumn{3}{|l|}{ Diagnosis } \\
\hline Hodgkin's lymphoma & 8 & 57.14 \\
\hline Diffuse large B cell lymphoma & 5 & 35.71 \\
\hline PTCL NOS & 1 & 7.14 \\
\hline \multicolumn{3}{|l|}{ Response to initial treatment } \\
\hline CR & 7 & 50.00 \\
\hline Refractory & 2 & 14.29 \\
\hline Progressive & 5 & 35.71 \\
\hline \multicolumn{3}{|l|}{ Response to relapse therapy } \\
\hline CR & 8 & 57.14 \\
\hline PD & 1 & 7.14 \\
\hline PR & 5 & 35.71 \\
\hline \multicolumn{3}{|l|}{ No. of treatment prior to transplan } \\
\hline 2 & 11 & 78.57 \\
\hline 3 & 2 & 14.29 \\
\hline 4 & 1 & 7.14 \\
\hline \multicolumn{3}{|l|}{ Disease status at time of transplant } \\
\hline CR & 8 & 57.14 \\
\hline PR & 6 & 42.86 \\
\hline
\end{tabular}

Abbreviations: CR, complete response; IPI, indicates international prognostic index; PD, progressive disease; $\mathrm{PR}$, partial response; PTCL NOS, peripheral T-cell lymphoma, not otherwise specified.
RDHAP was the most commonly used regimen as a second line treatment (11 patients, 78.5\%). Two patients (14.29\%) received RICE/ICE. Eight patients (57.14\%) were in CR at the time of transplant and six patients $(42.86 \%)$ were in partial response.

\section{Engraftment}

The median number of CD34+ cells infused was $5.95 \times 10^{6}$ cells per kilogram body weight (range, $3-17.2 \times 10^{6}$ cells per $\mathrm{kg}$ body weight). No engraftment failures were noted. The median number of days on GCSF was 24 (range, 13-40 days). The median time to neutrophil engraftment was 17 days (range, 14-29 days), and 24 days for platelet engraftment (range, 13-55 days) in our cohort. The average length of hospital stay was 20 days (range, 13-45 days).

\section{Non-hematological toxicity}

Electrolyte imbalances were a frequent complication in our patients. Serum potassium abnormalities were the most common with 7 patients (50\%) experiencing grade III (GIII) hyperkalemia and 7 patients (50\%) experiencing GI-II hypokalemia. Nine patients $(64.28 \%)$ experienced gastrointestinal (GI) hypomagnesemia. Liver dysfunction was also a common side effect in our cohort. Eight patients (57.14\%)

Table 2. Nonhematological toxicity.

\begin{tabular}{|c|c|c|}
\hline Adverse effect & Grade I-II & Grade III-V \\
\hline \multicolumn{3}{|l|}{ Electrolyte imbalances } \\
\hline Hypokalemia & $7(50 \%)$ & $7(50 \%)$ \\
\hline Hypomagnesemia & $10(71.4 \%)$ & $1(7.1 \%)$ \\
\hline \multicolumn{3}{|l|}{ Gastrointestinal toxicity } \\
\hline Mucositis & $5(35.7 \%)$ & $3(21.4 \%)$ \\
\hline Diarrhea & $8(57.1 \%)$ & $5(35.7 \%)$ \\
\hline Nausea/vomiting & $7(50 \%)$ & $1(7.1 \%)$ \\
\hline Abdominal pain & $3(21.4 \%)$ & \\
\hline Upper Gl bleed & $2(14.2 \%)$ & \\
\hline Lower Gl bleed & $1(7.1 \%)$ & \\
\hline \multicolumn{3}{|l|}{ Hepatotoxicity } \\
\hline Increase transaminases & & $1(7.1 \%)$ \\
\hline Increase ALP & $4(28.5 \%)$ & \\
\hline Hyperbilirubinemia & $4(28.5 \%)$ & $4(28.5 \%)$ \\
\hline \multicolumn{3}{|l|}{ Infectious complication } \\
\hline Febrile neutropenia & & $13(92.85 \%)$ \\
\hline Pneumonia & & $4(28.5 \%)$ \\
\hline Septic shock & & $4(28.5 \%)$ \\
\hline CMV infection & & $1(7.1 \%)$ \\
\hline \multicolumn{3}{|l|}{ Renal toxicity } \\
\hline Acute kidney injury & $5(35.7 \%)$ & $4(28.5 \%)$ \\
\hline \multicolumn{3}{|l|}{ Respiratory toxicity } \\
\hline Pleural effusion & & $3(21.4 \%)$ \\
\hline Respiratory failure & & $7(50 \%)$ \\
\hline \multicolumn{3}{|l|}{ Cardiotoxicity } \\
\hline Elevated troponins & $2(14.2 \%)$ & $2(14.2 \%)$ \\
\hline Arrhythmia & & $1(7.1 \%)$ \\
\hline \multicolumn{3}{|l|}{ CNS toxicity } \\
\hline Seizures & $1(7.1 \%)$ & $2(14.2 \%)$ \\
\hline
\end{tabular}


had mild hyperbilirubinemia and four (28.5\%) patients experienced GIII-IV toxicity. Nine patients (64.29) had acute kidney injury, among which eight patients (57.14\%) experienced GII-III toxicity. Three patients (21.4\%) had GIII-IV pleural effusion. Seven patients $(50 \%)$ developed respiratory compromise requiring $\mathrm{O}_{2}$ and NIV support. Eight (57.14\%) patients developed mucositis, of which five patients developed GI-II mucositis and three developed GIII mucositis. Gastrointestinal toxicity was seen in all of our patients. Twelve patients (85\%) developed GII-III diarrhea during their hospital stays. Cardiotoxicity was also observed in our cohort. Four patients exhibited increased troponin, of which two patients had GIII toxicity, and one patient had GIV arrhythmia.

Febrile neutropenia with GIII-IV severity was observed in $13(92.86 \%)$ patients. Among these patients, nine had fever of unknown origin, one had a lower respiratory tract infection (LRTI), and one had both LRTI and Staphylococcus species associated CLABSI infection. One patient had septicemia secondary to Staph epidermidis and $E$ coli. One patient had acinetobacter CMV pneumonitis as evidenced by tracheal cultures. Transplant-related mortality at day 100 was $28 \%$ (Table 2).

\section{Outcome analysis}

At the time of transplant, 8 patients $(57.14 \%)$ were in complete metabolic response (CMR) whereas 6 patients $(42.86 \%)$ were in PR. At day $+100,5$ patients (37\%) were in complete metabolic response on PET CT imaging, 3 (21\%) patients showed partial response, and 2 patients (14\%) had refractory disease. Transplant-related mortality at day 100 was $28 \%$. Among 8 patients who underwent ASCT in CMR, only 2 patients remained in $C R$ and were alive at the final follow-up. Among 6 patients who had partial response to ASCT, 5 patients were in CMR at evaluation and were alive at the last follow-up.

Patients with a low to intermediate IPI score exhibited better response and survival compared to patients who had

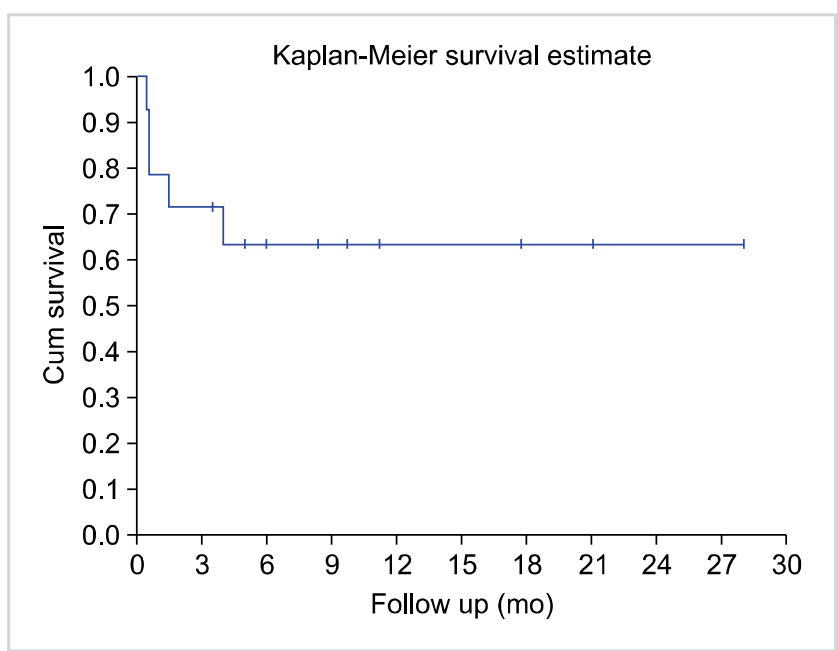

Fig. 1. Overall survival. a high IPI score. Nine patients who had low or low to intermediate IPI, 5 (55\%) and one out of three patients (33.33\%) with a high - intermediate IPI score patients in CMR at the last follow up.

The median time to follow-up was 5.5 months (15 days to $19 \mathrm{mo}$ ). At the final follow-up, 5 patients had died. Infections and subsequent systemic complications, particularly septic shock and hospital acquired pneumonia, were the most common cause of death in our cohort. Other causes included meningoencephalitis and CMV pneumonitis. At the final follow-up (median time), 7 patients (50\%) remained in complete response, 2 patients $(14.3 \%)$ had relapsed, and 5 patients (35.71\%) had died. The median disease-free survival was 5.96 months (range, 0.46-27.96 mo) and the median overall survival was 5.42 months (range, 0.46-27.96 mo) (Figs. 1, 2).

\section{DISCUSSION}

High-dose therapy followed by ASCT is the standard of care and the treatment of choice for eligible patients with chemosensitive, aggressive, relapsed NHL and HL. This treatment has been validated by the PARMA and the CORAL studies, which demonstrated a significantly higher survival rate of greater than $50 \%$ after high-dose chemotherapy and autologous bone marrow transplantation than after conventional chemotherapy [15]. Despite the improved survival rates provided by HDT/ASCT, applicability of this treatment to all relapsing patients is limited. Several factors impacted survival. Disease status is an important predictive factor for survival. Transplant-related morbidity and mortality is another important determinant of outcome.

The BEAM protocol has been the conditioning regimen of choice for ASCT since the initial report by Mills et al. [8] published in 1995. They reported that 5-year overall survival and progression-free survival rates were $41 \%$ and $35 \%$, respectively, with an early procedure-related mortality

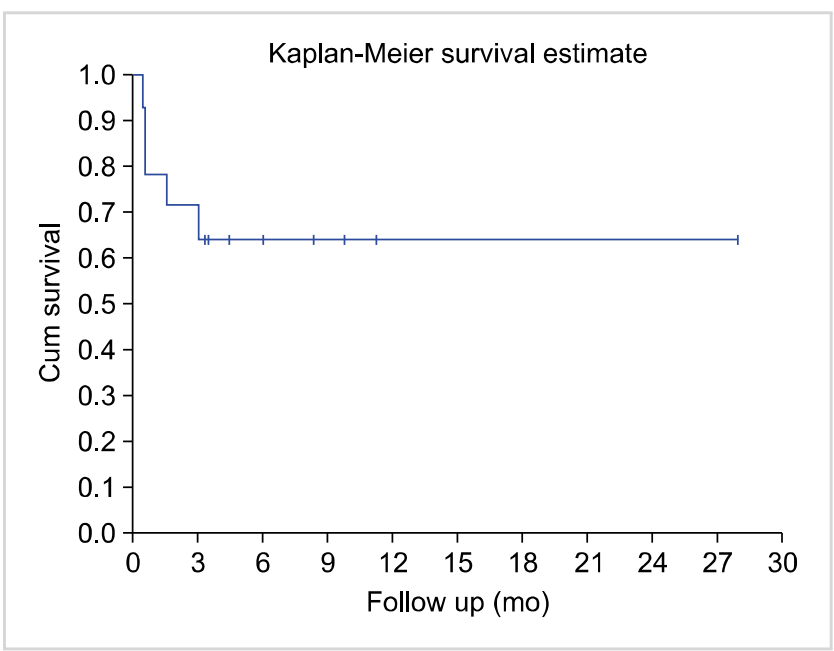

Fig. 2. Disease free survival. 
rate of $7 \%$. Over the years, improvement in patient selection, mobilization techniques, and supportive care has resulted in survival of greater than $70 \%$, and TRM rates less than $2 \%[16,17]$. A retrospective review published by Reid et al. [18] showed a trend toward better 3-year OS and PFS in response to an outpatient BEAM regimen compared to an inpatient BEAM regimen. This was also accompanied by a better toxicity profile. Furthermore mBEAM showed good tolerability, with the most frequently observed non-hematologic toxicity being oral mucositis, mainly of grade I/II (47\%). This tolerability was better than the BEAM regimen [11] or variants such as FEAM5 and BeEAM [19].

Late toxicities, however, remain a serious concern. Studies have revealed that non-infectious interstitial pneumonitis resulting from $\mathrm{BCNU}$-containing regimens was a serious complication, reported in $16-60 \%$ of the patients, with an associated mortality rate of $9 \%[8,20]$. BCNU-containing conditioning regimens have also been associated with an increased risk of non-relapse mortality when compared with other regimens [19-23]. Many regimens have been tested, and the BEAM regimen remains the standard of care despite its known serious side effects.

Unavailability of carmustine in many parts of the world has led to efforts to develop a conditioning regimen without carmustine. Visani et al. [14] reported results of a Phase I/II study using increasing doses of bendamustine $\left(160 \mathrm{mg} / \mathrm{m}^{2}\right.$, $180 \mathrm{mg} / \mathrm{m}^{2}$ and $200 \mathrm{mg} / \mathrm{m}^{2}$ given on day -7 and day -6) with fixed doses of etoposide, cytarabine, and melphalan (BeEAM regimen), administered as a preparative regimen for ASCT. Forty-three patients with resistant/relapsed HL $(\mathrm{N}=15)$ and NHL $(\mathrm{N}=28)$ were enrolled, with 9 in the phase I and 34 in the phase II studies. No patients experienced dose-limiting toxicity. TRM at day 100 was $0 \%$. The follow-up period at the time of publication was 18 months, with $81 \%$ of patients alive and disease-free at that time. Mucositis and GI toxicity were the most commonly observed side effects. No grade III/IV nephrotoxicity or cardiotoxicity was reported. These encouraging results led to further studies evaluating bendamustine as a component of the conditioning regimen.

In a large French multicenter study (Lysa group), bendamustine was administered at a median dose of 191 $\mathrm{mg} / \mathrm{m}^{2} /$ day (range, $50-250 \mathrm{mg} / \mathrm{m}^{2} /$ day) on days 6 and 7 with standard doses of etoposide, melphalan, and cytarabine. The results of this study highlighted early toxicities with bendamustine which may have been underrepresented in the Visani study due to small sample size. One hundred seven (28\%) patients developed grade I-IV nephrotoxicity. Patients who developed ARF were also more likely to experience colitis, pneumonitis, cardiac arrhythmias, intensive care admission, and death. Multivariate analysis showed that creatinine level at day 1 , bendamustine dose $>160 \mathrm{mg} / \mathrm{m}^{2}$, and age were independent prognostic factors for ARF. Ten percent of patients needed intensive care management, and the toxic death rate was estimated at 5\% [24]. Garciaz et al. [25] reported increased nephrotoxicity in response to BeAM in their patients. Nine (31\%) patients developed early renal toxicity before the day of reinfusion, of which four (14\%) patients experienced grade 3-4 renal impairment. At day 30, the cumulative incidence of nephrotoxicity was $48 \%$ (17\% of grade $3-4)$, whereas in the BEAM cohort, renal impairment was only observed in $7 \%$ of patients $(5 \%$ of grade $3-4, P<0.001)$. As also noted by the Lysa group, patients who developed nephrotoxicity were more likely to need admission to the intensive care unit [24\% of patients in the Lysa group vs. $10 \%$ of patients in the BEAM group $(P=0.07)]$. Cardiotoxicity was also more common in the BeAM group, with grade 2-3 cardiac toxicity observed in $10 \%$ of patients (vs. $2 \%$ in the BEAM cohort, $P=0.02$ ).

Our study showed a similar toxicity profile. Twenty-eight percent of our patients developed grade III-IV nephrotoxicity. In addition, we saw a higher incidence of grade II-IV colitis and hepatotoxicity than reported in previous studies. Septic shock and respiratory failure were observed in $28 \%$ and $50 \%$ of patients, respectively. Four patients $(28 \%)$ in our cohort died in the early transplant period as a result of septic shock and its complications. The cause of the higher incidence of toxic death compared to that in other similar studies is unclear and needs further examination. One hypothesis is that higher doses of bendamustine were responsible for increased toxicity. The Lysa group reported increased toxicity at doses greater than $160 \mathrm{mg} / \mathrm{m}^{2}$. In our protocol, we used $150 \mathrm{mg} / \mathrm{m}^{2}$ of bendamustine at days 4 and 5. Despite this dose reduction, we observed significant toxicities. Therefore, it is our opinion the appropriate dose and schedule of bendamustine requires further study.

In conclusion, in our retrospective analysis, we reported a high incidence of renal and cardiac toxicity and an unusually high transplant-related mortality of $28 \%$ with bendamustine in the conditioning regimen for autologous stem cell transplant. Therefore, bendamustine should be used with caution. Prospective comparative studies are needed to confirm the appropriate dose and schedule of bendamustine, and to further evaluate the toxicities noted in retrospective studies before it is routinely adopted in clinical practice.

\section{Authors' Disclosures of Potential Conflicts of Interest}

No potential conflicts of interest relevant to this article were reported.

\section{REFERENCES}

1. Kuruvilla J. Standard therapy of advanced Hodgkin lymphoma. Hematology Am Soc Hematol Educ Program 2009;2009:497-506.

2. Kewalramani T, Zelenetz AD, Nimer SD, et al. Rituximab and ICE as second-line therapy before autologous stem cell transplantation for relapsed or primary refractory diffuse large B-cell lymphoma. Blood 2004;103:3684-8.

3. Ardeshna KM, Kakouros N, Qian W, et al. Conventional second-line salvage chemotherapy regimens are not warranted in patients with malignant lymphomas who have progressive disease 
after first-line salvage therapy regimens. Br J Haematol 2005; 130:363-72.

4. Bierman PJ, Armitage JO. Looking back (and ahead) at salvage treatment for non-Hodgkin lymphoma. Oncology (Williston Park) 2009;23:619.

5. Philip T, Guglielmi C, Hagenbeek A, et al. Autologous bone marrow transplantation as compared with salvage chemotherapy in relapses of chemotherapy-sensitive non-Hodgkin's lymphoma. N Engl J Med 1995;333:1540-5.

6. Reddy NM, Oluwole O, Greer JP, Engelhardt BG, Jagasia MH, Savani BN. Outcomes of autologous or allogeneic stem cell transplantation for non-Hodgkin lymphoma. Exp Hematol 2014;42:39-45.

7. Akhtar S. High dose chemotherapy and autologous stem cell transplantation in relapsed or refractory Hodgkin lymphoma: Emerging questions, newer agents, and changing paradigm. Hematol Oncol Stem Cell Ther 2017;10:272-6.

8. Mills W, Chopra R, McMillan A, Pearce R, Linch DC, Goldstone AH. BEAM chemotherapy and autologous bone marrow transplantation for patients with relapsed or refractory nonHodgkin's lymphoma. J Clin Oncol 1995;13:588-95.

9. Puig N, de la Rubia J, Remigia MJ, et al. Morbidity and transplant-related mortality of $\mathrm{CBV}$ and BEAM preparative regimens for patients with lymphoid malignancies undergoing autologous stem-cell transplantation. Leuk Lymphoma 2006;47: 1488-94.

10. Liu HW, Seftel MD, Rubinger M, et al. Total body irradiation compared with BEAM: Long-term outcomes of peripheral blood autologous stem cell transplantation for non-Hodgkin's lymphoma. Int J Radiat Oncol Biol Phys 2010;78:513-20.

11. Leoni LM, Bailey B, Reifert J, et al. Bendamustine (Treanda) displays a distinct pattern of cytotoxicity and unique mechanistic features compared with other alkylating agents. Clin Cancer Res 2008;14:309-17.

12. Friedberg JW, Cohen $\mathrm{P}$, Chen $\mathrm{L}$, et al. Bendamustine in patients with rituximab-refractory indolent and transformed non-Hodgkin's lymphoma: results from a phase II multicenter, single-agent study. J Clin Oncol 2008;26:204-10.

13. Brugger W, Ghielmini M. Bendamustine in indolent non-Hodgkin's lymphoma: a practice guide for patient management. Oncologist 2013;18:954-64.

14. Visani G, Malerba L, Stefani PM, et al. BeEAM (bendamustine, etoposide, cytarabine, melphalan) before autologous stem cell transplantation is safe and effective for resistant/relapsed lymphoma patients. Blood 2011;118:3419-25.

15. Gisselbrecht C, Glass B, Mounier N, et al. Salvage regimens with autologous transplantation for relapsed large B-cell lymphoma in the rituximab era. J Clin Oncol 2010;28:4184-90.

16. Caimi PF, William BM, Silva Rondon $\mathrm{CH}$, et al. Comparison of 2 carmustine-containing regimens in the rituximab era: excellent outcomes even in poor-risk patients. Biol Blood Marrow Transplant 2015;21:1926-31.

17. Czyz A, Lojko-Dankowska A, Dytfeld D, et al. Prognostic factors and long-term outcome of autologous haematopoietic stem cell transplantation following a uniform-modified BEAM-conditioning regimen for patients with refractory or relapsed Hodgkin lymphoma: a single-center experience. Med Oncol 2013;30:611.

18. Reid RM, Baran A, Friedberg JW, et al. Outpatient administration of BEAM conditioning prior to autologous stem cell transplantation for lymphoma is safe, feasible, and cost-effective. Cancer Med 2016;5:3059-67.

19. Galieni P, Troiani E, Bigazzi C, et al. Modified BEAM as conditioning regimen for lymphoma patients undergoing autologous hematopoietic stem cell transplantation. Bone Marrow Transplant 2018;53:91-3.

20. van Besien K, Tabocoff J, Rodriguez M, et al. High-dose chemotherapy with BEAC regimen and autologous bone marrow transplantation for intermediate grade and immunoblastic lymphoma: durable complete remissions, but a high rate of regimen-related toxicity. Bone Marrow Transplant 1995;15: 549-55.

21. Gribben JG, Linch DC, Singer CR, McMillan AK, Jarrett M, Goldstone AH. Successful treatment of refractory Hodgkin's disease by high-dose combination chemotherapy and autologous bone marrow transplantation. Blood 1989;73:340-4.

22. Visani G, Picardi P, Tosi P, et al. Autologous stem cell transplantation for aggressive lymphomas. Mediterr J Hematol Infect Dis 2012;4:e2012075.

23. Sun CL, Francisco L, Baker KS, Weisdorf DJ, Forman SJ, Bhatia S. Adverse psychological outcomes in long-term survivors of hematopoietic cell transplantation: a report from the Bone Marrow Transplant Survivor Study (BMTSS). Blood 2011;118: 4723-31.

24. Chantepie S, Tchernonog E, Peyrade F, et al. Bendamustine-based (BeEAM) conditioning before autologous stem cell transplantation: result of a French multicenter study of 386 patients from Lysa Centers. Blood (ASH Annual Meeting Abstracts) 2016;128(Suppl):abst 3450.

25. Garciaz S, Coso D, Schiano de Collela JM, et al. Bendamustinebased conditioning for non-Hodgkin lymphoma autologous transplantation: an increasing risk of renal toxicity. Bone Marrow Transplant 2016;51:319-21. 